\title{
Piammiano (Statonia ?)
}

Prospections géophysiques sur le site de Piammiano (Statonia?)

Vincent Jolivet et Claire Joncheray

\section{(2) OpenEdition \\ Journals}

Édition électronique

URL : http://journals.openedition.org/cefr/959

DOI : $10.4000 /$ cefr.959

ISSN : 2282-5703

Éditeur

École française de Rome

Référence électronique

Vincent Jolivet et Claire Joncheray, «Piammiano (Statonia?) », Chronique des activités archéologiques de l'École française de Rome [En ligne], Italie centrale, mis en ligne le 23 mai 2013, consulté le 20 avril 2019. URL : http://journals.openedition.org/cefr/959; DOI : 10.4000/cefr.959

Ce document a été généré automatiquement le 20 avril 2019

(c) École française de Rome 


\section{Piammiano (Statonia ?)}

Prospections géophysiques sur le site de Piammiano (Statonia?)

\section{Vincent Jolivet et Claire Joncheray}

Situé sur le territoire de la commune de Bomarzo, au nord du Latium, dans la province de Viterbe, le site de Piammiano (fig. 1), fouillé depuis le début du XIX ${ }^{e}$ siècle, a fait l'objet en 2012 d'une campagne de prospections géophysiques placée sous la responsabilité de Vincent Jolivet avec la participation de Claire Joncheray.

Fig. 1 - Le plateau de Piammiano, emplacement supposé du site étrusque et de la praefectura romaine de Statonia.

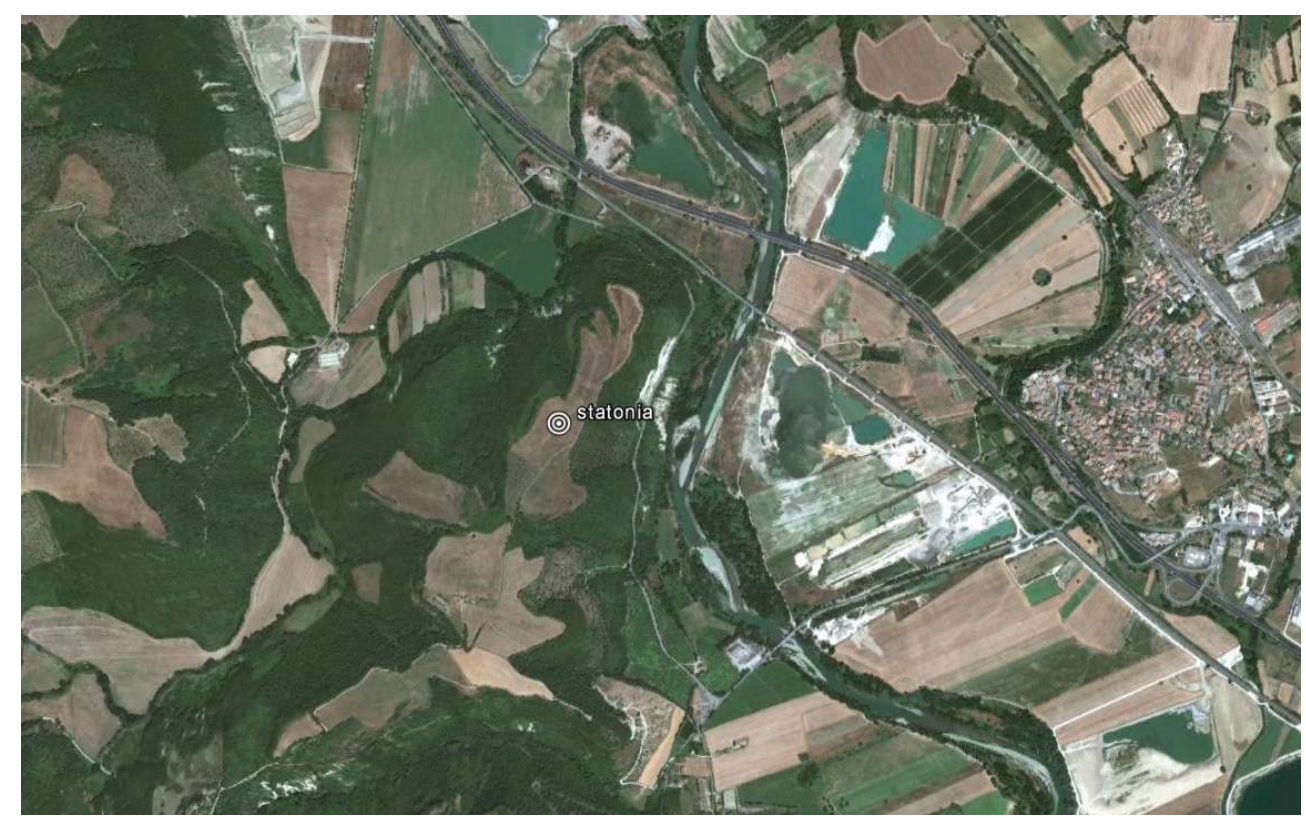

2 Financée par un Plan Pluri-Formation du département des Sciences de l'Antiquité de l'École normale supérieure de Paris, cette campagne a été réalisée en collaboration avec la Soprintendenza per i Beni Archeologici dell'Etruria Meridionale (sous le contrôle de la dott.ssa Maria Letizia Arancio) et avec l'École française de Rome. Nous remercions la 
municipalité de Bomarzo pour l'hospitalité qu'elle nous a accordée au cours de nos différents séjours sur place.

3 L'intérêt pour ce site s'inscrit dans une réflexion plus générale sur les limites du territoire de Tarquinia, menée à partir de l'étude et de la publication en cours du site étruscoromain de Musarna, fouillé par l'École française de Rome entre 1983 et 2003. Les recherches récentes sur le site de Statonia ${ }^{1}$, dont nous savons par Vitruve qu'il faisait partie de ce territoire, invitent en effet à le localiser non plus à l'ouest du lac de Bolsena, mais sur la rive droite du Tibre, à la hauteur approximative de la cité ombrienne d'Amelia. On peut donc penser que le territoire de la grande métropole étrusque méridionale ne s'achevait pas vers l'Est au niveau des monts Ciminiens, selon la reconstruction traditionnelle, mais s'étendait jusqu'au Tibre, ce que le dossier archéologique, du moins pour l'époque hellénistique, semble largement corroborer².

Dans cette zone, la colline de Piammiano, qui domine le Tibre à $2,5 \mathrm{~km}$ au nord-est de Bomarzo, à une altitude moyenne de $180 \mathrm{~m}$, est apparue d'emblée comme le lieu le plus susceptible d'avoir été le siège du site étrusque et de la praefectura romaine qui en a pris la suite dans le courant du III siècle av. J.-C., et qui est encore attestée à l'époque augustéenne: en surface, les témoignages d'occupation d'époque historique, compris entre le VII siècle av.J.-C. et le $\mathrm{VI}^{\mathrm{e}}$ siècle ap. J.-C., y occupent une aire de l'ordre de 15 hectares $^{3}$. Le site a fait l'objet de campagnes de prospections magnétiques, de prospections de surface et de fouilles organisées conjointement par les universités de Sienne (prof. Emanuele Papi) et de Berkeley (prof. Theodor Peña) entre 1998 et 2000, dont les résultats n'ont été publiés à ce jour que sous forme d'un bref compte rendu ${ }^{4}$; ces travaux, menés dans quatre secteurs différents, ont notamment permis de mettre au jour dans la partie centrale du plateau, sur une superficie de $600 \mathrm{~m}^{2}$ environ, des rues et un quartier d'habitation d'époque hellénistique, romaine et médiévale ${ }^{5}$.

5 L'objectif de l'opération était donc de chercher à établir, par l'intermédiaire d'une prospection géophysique aussi complète que possible en fonction des crédits disponibles, confiée à la société Geocarta (www.geocarta.fr), si le sous-sol recelait effectivement des vestiges susceptibles de correspondre à la vaste agglomération que devait représenter cette praefectura, avec ses monuments publics et ses quartiers d'habitation, et à préciser les caractéristiques de l'urbanisme de la cité antique.

6 Compte tenu des caractéristiques géologiques et archéologiques du terrain, la prospection a été réalisée en $\mathrm{ARP}^{\odot}$ (méthode électrique tractée) sur une superficie de 7 hectares, les 23 et 24 avril 2012, par Giovanni Bitella (société SOING) ; le secteur d'intervention, correspondant approximativement à la moitié de l'extension supposée du site, a été choisi en raison de sa position centrale par rapport à celle-ci, en laissant, au nord et au sud, deux zones inexplorées. Deux secteurs, séparés par un chemin de terre, apparaissent clairement différenciés sur les cartes de résistivité électrique, dont la publication définitive devrait faire l'objet d'une étude plus circonstanciée, intégrant les résultats des recherches effectuées précédemment sur le site (fig. 2). 
Fig. 2 - Le plateau de Piammiano. Carte géophysique générale.

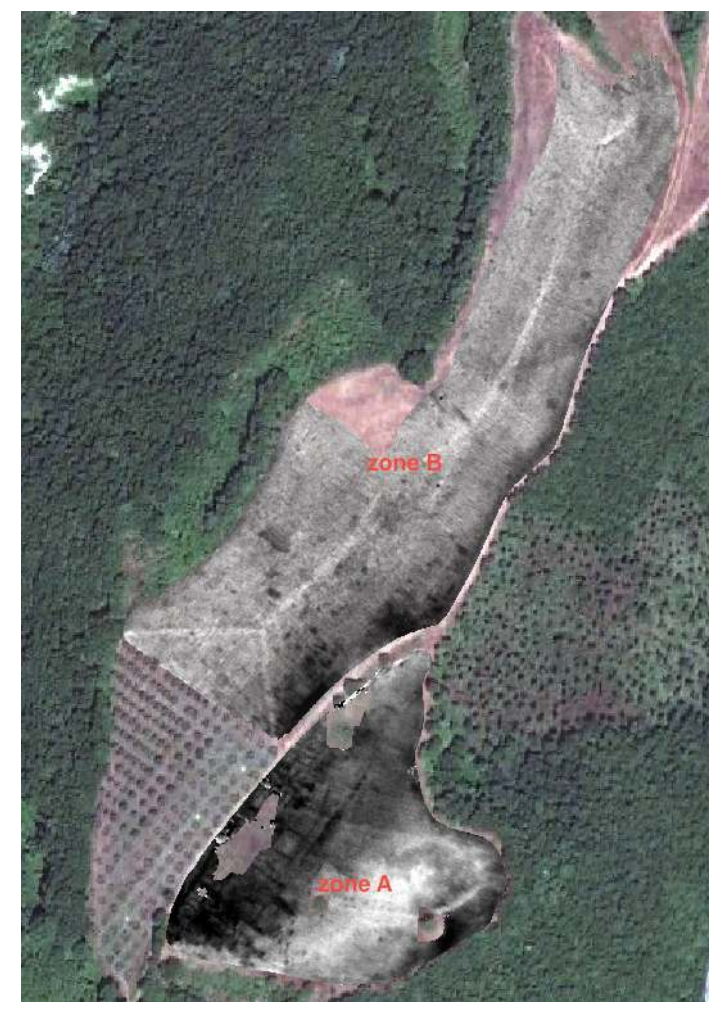

7 Au sud-est de la zone prospectée (zone A), sur le tiers environ de sa superficie totale, dans le secteur fouillé par l'équipe italo-américaine, la carte met clairement en évidence de nombreuses anomalies linéaires et le plus souvent perpendiculaires les unes aux autres, organisées en fonction de deux axes principaux, nord-est/sud-ouest et nord-ouest/sudest, et clairement situées dans le prolongement des structures encore visibles sur le site, qui pourront contribuer à leur interprétation; mais la trace de ces vestiges disparaît dans toute la moitié orientale de cette zone. Dans la partie centrale et septentrionale du secteur étudié (zone B), en revanche, toute la partie prospectée ne présente, sauf au sudest, dans le prolongement de la zone A, aucun témoignage bien clair d'intervention anthropique, à l'exception de traces en relation avec l'exploitation agricole du champ ; toutefois, l'extrémité nord du plateau présente à nouveau quelques traces de constructions, ainsi qu'une trace semi-circulaire qui pourrait être en liaison avec le dispositif défensif du site.

Sur les 7 hectares prospectés, une petite partie seulement (environ 1 ha) a donc révélé la présence de structures enfouies. Seule une opération de fouille archéologique pourrait permettre d'interpréter correctement cette lacune et d'établir si les travaux agricoles ont porté à la destruction complète des vestiges de la cité antique, ou si le centre de l'habitat doit être recherché plutôt dans la partie du plateau située plus au Sud, qui n'a pas été prospectée cette année, et où sont encore visibles les vestiges d'une grande citerne romaine ; mais il n'est pas non plus exclu, sur ces bases, qu'il faille rechercher Statonia à un autre emplacement, au sein de cette même région tibérine. 


\section{BIBLIOGRAPHIE}

Jolivet à paraître $=\mathrm{V}$. Jolivet, Nouvelles frontières, nouveaux horizons. Les contours changeants $d u$ territoire de Tarquinia, dans Mélanges Dominique Briquel, à paraître.

Gasperoni - Scardozzi, 2010 = T. Gasperoni, G. Scardozzi, Bomarzo, Mugnano, Bassano in Teverina, Carta archeologica d'Italia. Contributi, Viterbe, 2010, p. 316-328.

Gliozzo 2007 = E. Gliozzo, The Distribution of Bricks and Tiles in the Tiber Valley. The Evidence of Piammiano (Bomarzo, Viterbo), dans E. Papi (dir.), Supplying Rome and the Empire, Portsmouth, 2007, p. 59-72 (JRA, suppl. 69).

Munzi 1995 = M. Munzi, La nuova Statonia, dans Ostraka, 4, 1995, p. 285-299.

Munzi, 1998 = M. Munzi, I praedia statoniensia dei Sestii : rinvenimenti epigrafici a Piammiano (Bomarzo), dans Ostraka, 7, 1998, p. 85-92.

Papi - Peña, 2000 = E. Papi, J. T. Peña, The Statonia Project : Report on the 1998 and 1999 Field Seasons, dans AJA, 104, 2000, p. 346.

Stanco 1994 = E. A. Stanco, La localizzazione di Statonia : nuove considerazioni in base alle antiche fonti, dans MEFRA, 106-1, 1994, p. 247-258.

\section{NOTES}

1. Stanco 1994, p. 247-258 ; Munzi 1995, p. 285-299 ; Munzi, 1998, p. 85-92.

2. Jolivet à paraître.

3. Voir maintenant l'excellente carte archéologique figurant dans Gasperoni - Scardozzi, 2010, p. 316-328.

4. Papi - Peña, 2000, p. 346. Voir aussi Gliozzo 2007, p. 59-72.

5. Nous remercions Emanuele Papi et Theodor Peña pour les informations inédites qu'ils ont bien voulu nous fournir sur les travaux, en cours de publication, qu'ils ont réalisés sur le site.

\section{INDEX}

Mots-clés : Statonia, Tarquinia, étrusque, Étrurie, urbanisme, cité antique institutions Soprintendenza archeologica per i Beni Archeologici dell'Etruria meridionale, AOROC (UMR 8546 CNRS-ENS), École normale supérieure de Paris, École française de Rome 


\section{AUTEURS}

\section{VINCENT JOLIVET}

CNRS, AOROC (UMR 8546 CNRS-ENS) - vincent_jolivet[at]libero.it

\section{CLAIRE JONCHERAY}

Centre Camille Jullian (Université Aix-Marseille / CNRS) - claire.joncheray[at]free.fr 